\title{
Human genetics of infectious diseases: between proof of principle and paradigm
}

\author{
Alexandre Alcaïs, 1,2 Laurent Abel,1,2,3 and Jean-Laurent Casanova1,2,3,4
}

\begin{abstract}
${ }^{1}$ Laboratory of Human Genetics of Infectious Diseases, Necker Branch, INSERM U550, Paris, France. 2University Paris Descartes, Necker Medical School, Paris, France. ${ }^{3}$ Laboratory of Human Genetics of Infectious Diseases, Rockefeller Branch, The Rockefeller University, New York, New York, USA. ${ }^{4}$ Pediatric Hematology-Immunology Unit, Necker Hospital, Paris, France.
\end{abstract}

\begin{abstract}
The observation that only a fraction of individuals infected by infectious agents develop clinical disease raises fundamental questions about the actual pathogenesis of infectious diseases. Epidemiological and experimental evidence is accumulating to suggest that human genetics plays a major role in this process. As we discuss here, human predisposition to infectious diseases seems to cover a continuous spectrum from monogenic to polygenic inheritance. Although many studies have provided proof of principle that infectious diseases may result from various types of inborn errors of immunity, the genetic determinism of most infectious diseases in most patients remains unclear. However, in the future, studies in human genetics are likely to establish a new paradigm for infectious diseases.
\end{abstract}

\section{Introduction}

The determinism of human infectious diseases is still widely misunderstood, with these diseases commonly thought to be purely infectious. As exposure to a microbial agent is obviously required for infection and disease to occur, infectious diseases are often regarded as textbook proof-of-principle examples of purely environmental diseases. However, following the publication of Pasteur's microbial theory of disease from 1865 to 1870 (1), the most important unresolved question concerned the astounding level of clinical variability between individuals in a population infected with the same microbe (Figure 1). Clinical features and outcome vary greatly from patient to patient. Speculation about and investigation of natural variability in the development of infectious diseases were boosted between 1911 and 1917 by Charles Nicolle's discovery of the coexistence of symptomatic and asymptomatic infections in human populations (2). Various theories have been proposed to account for this heterogeneity $(1,3)$. In particular, epidemiological evidence has accumulated, since the 1930s, that human genetic factors play a particularly important role in immunodeficiency and susceptibility to infectious diseases (4-7). The first evidence supporting the genetic theory of infectious diseases came from observations of ethnic and familial aggregation of both rare and common infections, which even followed a Mendelian (monogenic) pattern of inheritance in some kindreds (8). Followup studies of adoptive children also showed that predisposition to infectious diseases was largely inherited, paradoxically more so than that to diseases associated with less-well-known environmental risk factors, such as cancer (9). Finally, the concordance rate of infectious diseases is higher in monozygotic twins than in dizygotic twins, implicating host genetic background in susceptibility to these diseases $(6,8)$.

The field of the human genetics of infectious diseases entered the molecular and cellular era in the early 1950s, with a series of landmark discoveries. These included the discovery by Ogden Bruton of individuals with X-linked agammaglobulinemia (XLA) (10), a rare X-linked disorder caused by mutations in Bruton agammaglobulinemia tyrosine kinase that result in a lack of mature B cells, and by Anthony Allison of the protective role of

Conflict of interest: The authors have declared that no conflict of interest exists. Citation for this article: J. Clin. Invest. 119:2506-2514 (2009). doi:10.1172/JCI38111. the sickle cell trait against the severe forms of malaria caused by Plasmodium falciparum (11). According to the dominant paradigm developed following these groundbreaking discoveries, predisposition to infectious diseases segregates in either a Mendelian or polygenic pattern of inheritance. An ever-growing number of rare Mendelian inborn errors of immunity associated with multiple infections, often referred to as conventional primary immunodeficiencies (PIDs), have since been reported $(12,13)$. For more common infectious diseases (such as malaria), genetic predisposition is thought to involve many genes, each of which has a modest effect (14). However, the distinction between Mendelian predisposition in individuals with rare infections (one gene, multiple infections) and complex predisposition in populations with common infections (one infection, multiple genes) has become somewhat blurred in recent years (4). Rare nonconventional PIDs conferring predisposition to a single type of infection in otherwise healthy individuals are increasingly being recognized (one gene, one infection) $(15,16)$. Mendelian resistance to more virulent pathogens has also been described (e.g., refs. 17, 18) - implying common Mendelian susceptibility in the rest of the population. Moreover, the so-called polygenic susceptibility may primarily reflect the impact of a predominant gene, often referred to as a major gene (19). In fact, the commonly diffused concept of polygenic inheritance remains to be demonstrated, at least at the individual level. In this Review, we provide an overview of the various types of genetic susceptibilities underlying human infectious diseases, illustrated by key examples (Figure 2).

\section{Mendelian predisposition to multiple infections}

Perhaps the most compelling evidence that human genetics may indeed determine the development of infectious diseases arises from PIDs, first described in the late 1940s and early 1950s, following the advent of antibiotics. Although most children were cured by these new drugs, it soon became clear that a minority had recurrent and multiple infections, a finding that it would have been difficult to document earlier. Moreover, some had immunological abnormalities, such as agammaglobulinemia (a lack of serum Ig), the observation of which also required technical progress $(10,20)$. More than 200 PIDs have since been clinically described, and the genetic etiology of over 150 of these diseases has been elucidated $(12,13)$. Typically, these disorders are individ- 
A Pasteur's pedigree

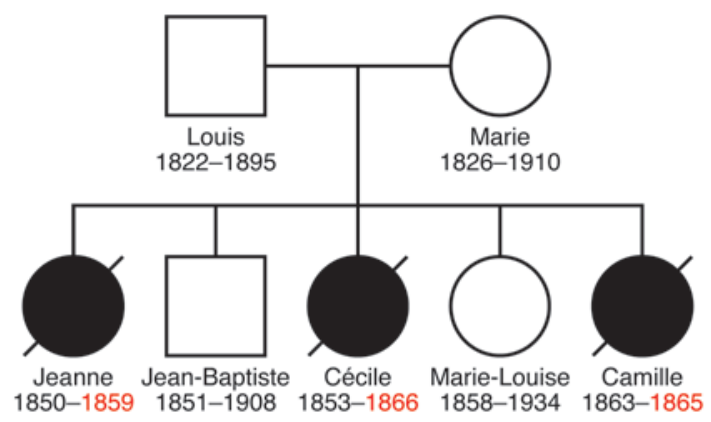

B Darwin's pedigree
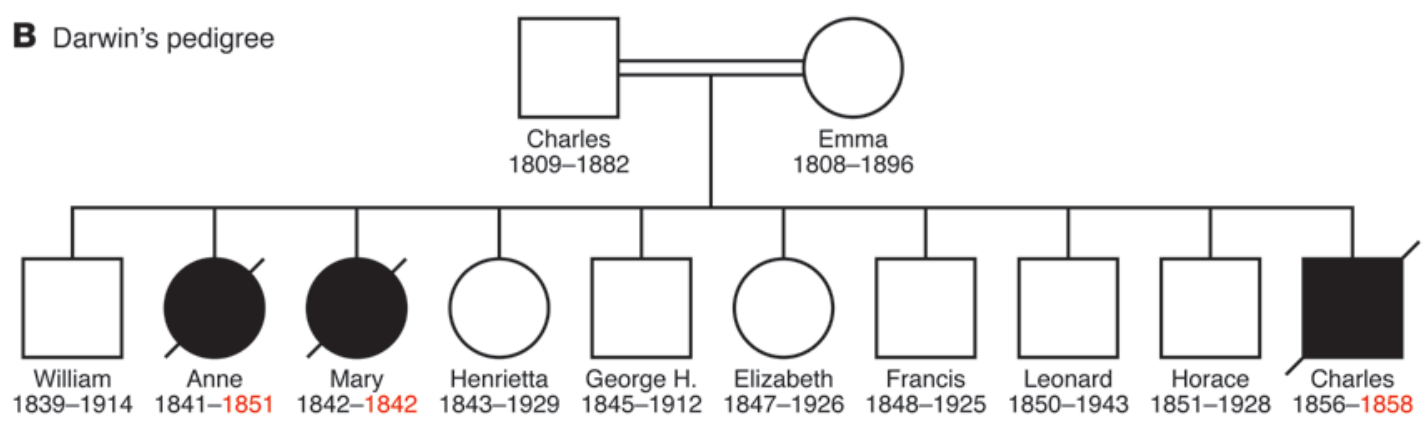

Figure 1

The pedigrees of Pasteur and Darwin and the genetic theory of infectious diseases. (A) Louis Pasteur, the founder of the microbial theory of disease, lost three young daughters to "fever" between 1859 and 1866. A few years later, in 1870, he discovered that microbes caused disease in silkworms (95), paving the way for a general microbial theory of disease. Retrospectively, it is clear that his daughters died of infectious diseases. (B) Charles Darwin, the founder of the theory of natural selection, also lost three children to infectious diseases. Charles and Emma Darwin were first cousins. These two illustrious families are representative of most families worldwide and throughout history, until recent improvements in hygiene and the advent of vaccines and antibiotics, which resulted from the microbial theory. Prior to these medical advances, it was not uncommon for at least half the siblings in a family to die of infection. The microbial theory of disease identified the microbial cause of disease but did not resolve the question of intrafamilial clinical heterogeneity in families exposed to the same microbial environment. As illustrated in the pedigrees of Pasteur and Darwin, some children survived until adulthood, despite probable exposure to at least one of the microbes that killed their other siblings. It is possible that the children who died carried a Mendelian trait predisposing them to infectious diseases, or at least had some form of genetic predisposition to such diseases.

ually rare, Mendelian, and confer predisposition to multiple infectious diseases - the number and nature of which differ among individuals. They affect immune responses in various ways, typically, but not exclusively, involving hematopoietic cells. Typical forms of PID include severe congenital neutropenia (SCN), a lack of polymorphonuclear leukocytes (PMNs) (21); XLA, a lack of B cells (22); and SCID, a lack of T cells (23). These disorders contributed to the definition of the function of the lost leukocyte subsets in host defense. Children with XLA, like those with SCN, experience multiple infections with "extracellular" pathogens, but the range of pathogens in the two conditions only partly overlaps. These experiments of nature revealed the role of Ig and granulocytes in host defense, long before the corresponding mutant mice became available. Children with SCID present with multiple infectious diseases caused by viruses, bacteria, fungi, and parasites in the first year of life, typically beginning between the ages of two and three months. A broad range of weakly virulent microorganisms can kill such patients. SCID is invariably fatal when it follows its natural course, even in specialist hospitals, typically in the first year of life. SCID patients progressively succumb to the succession of infections, even if treated with anti-infectious agents. These three disorders neatly illustrate the life-threatening impact of Mendelian genetics in terms of infectious diseases.
Each of these three disorders was historically associated with a distinct medical revolution. Specifically, they each led to the development of a completely novel line of treatment, based not on antibiotics but on the restoration of immunity - renewing interest in the line of research that had dominated human medicine from Louis Pasteur's development of vaccination and Charles Richet's and Emil von Behring's development of serotherapy. Life-saving substitution with gammaglobulins (later IgG) was first given to children with XLA in 1950 (20). Recombinant G-CSF was not initially used in patients with SCN, but it soon seemed to be of considerable benefit to these children, with a first report in 1989 (24). Both G-CSF and IgG are now used widely and safely, with no major side effects. By contrast, SCID patients have proven interesting in terms of the information they provide about both the life-saving efficacy and the risks of death associated with novel treatments. Indeed, both HSC transplantation (HSCT) and gene therapy had their first successes and failures in patients with SCID. The first successful HSCT in humans was performed in 1968 , by Richard Gatti and Robert Good, in a child with SCID $(25,26)$. Despite the rarity of graft rejection, it was soon realized that children undergoing HSCT could die of graft-versus-host disease or severe infections. More than 30 years later, a series of ten children with SCID were treated by gene therapy by Alain 


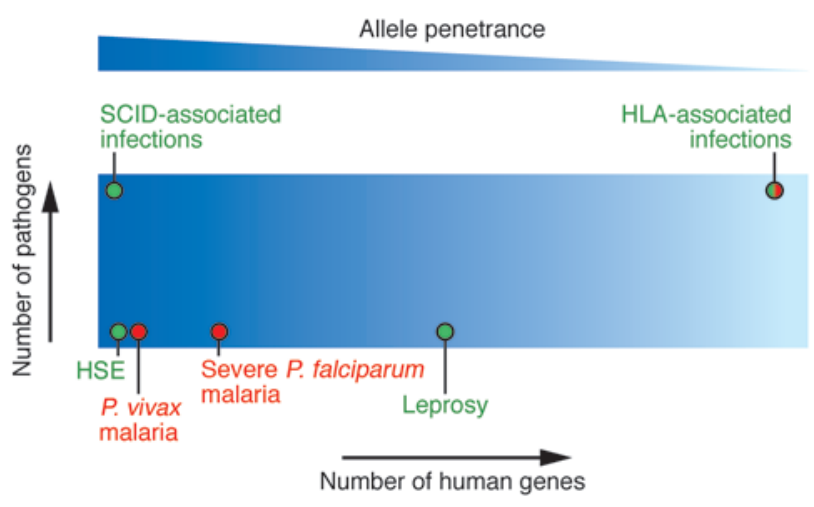

Figure 2

Schematic representation of the continuous genetic models underlying human infectious diseases. The spectrum of genetic susceptibilities predisposing individuals to infectious diseases is summarized. Different situations may be distinguished according to the number of genes with an additive impact on genetic susceptibility (in green) or resistance (in red), the marginal effect of each of these genes, and the number of pathogens to which the individual is susceptible. Six textbook examples are shown: SCID-associated infections (a unique gene with complete penetrance conferring predisposition to a large spectrum of infectious agents); HSE (a single gene with high penetrance conferring predisposition to a single infectious agent); malaria caused by $P$. vivax (a single gene with high penetrance conferring resistance to a single infectious agent); severe malaria caused by $P$. falciparum (a small number of genes with $\mathrm{HbS}$ conferring resistance to the disease); leprosy (a small number of genes with intermediate penetrance conferring predisposition to a single infectious agent); diseases in which HLA alleles have been shown to play a role (HLA-associated infections). Examples of common infectious diseases favored by multiple predisposing alleles in a given individual (truly multigenic inheritance) may be revealed by future GWA studies.

Fischer in France (27). Remarkably, nine of the ten children with SCID developed normal numbers of functional $T$ cells, which were maintained for up to eight years in the first child treated (23). However, a few years after gene therapy, four of the nine children developed acute $T$ cell leukemia $(23,28,29)$. Insertional mutagenesis and aberrant transcription of the LIM domain-only 2 (LMO2) oncogene were involved in leukemogenesis in most of these patients $(28,29)$. Sadly, one of these four patients with leukemia has died, despite chemotherapy and HSCT. One of the ten patients treated with a similar protocol in the United Kingdom has also since developed leukemia $(30,31)$. However, this innovative care of patients with SCID provided proof of principle that both HSCT and gene therapy may be clinically beneficial.

\section{Mendelian predisposition to single infections}

It gradually became clear that not all PIDs confer predisposition to multiple infections, or even recurrent infections. An increasing number of disorders (summarized in Table 1) are known to confer Mendelian predisposition to a single type of infection (15, 16). Epidermodysplasia verruciformis (EV), an extremely rare disorder that predisposes to skin warts and cancer, was described clinically as early as 1922 . The genetic origin of this syndrome was proposed in 1933, and its viral etiology (human papillomavirus [HPV]) was documented in 1946 (32). It was therefore probably the first PID ever described - preceding even XLA - as it corresponds to a monogenic predisposition to infection. The lack of an overt immunological phenotype and the extremely narrow spectrum of infections, limited to those caused by certain oncogenic papillomaviruses, probably precluded the use of the term PID at the time of its identification. The first two EV-causing genes, EVER1 and EVER2, were described in $2002(33,34)$. An X-linked form of predisposition to lethal infection by EBV was reported in 1975 (35), and two causal genes have been identified to date, SLAM-associated protein $(S A P)$ and X-linked inhibitor of apoptosis (XIAP) (36). Mendelian predispositions to a single type of bacterial infection have also been described. Patients with either X-linked properdin deficiency or autosomal recessive defects in the terminal components of the complement cascade (C5-C9), which form the membrane attack complex, display a selective predisposition to invasive meningococcal disease (16). A predisposition to invasive pneumococcal disease also led to the identification of patients with IL-1 receptor-associated kinase 4 (IRAK-4) deficiency $(37,38)$, as well as patients with MyD88 deficiency (39). The most thoroughly characterized of these syndromes is probably Mendelian susceptibility to mycobacterial diseases (MSMD). Despite the clinical description of MSMD in the 1950s, it was not until 1996 that the first genetic etiology of this syndrome - IFN- $\gamma$ R 1 deficiency - was identified $(40,41)$. In the last ten years, up to 13 genetic defects affecting six genes have been reported to cause $\operatorname{MSMD}(42,43)$. The six genes are physiologically related: they all encode proteins involved in IL-12/IL-23-dependent, IFN- $\gamma$-mediated immunity (42-44). Interestingly, as in individuals with either IRAK-4 or MyD88 deficiency, the clinical status of individuals with either IL-12p40 or IL-12R $\beta 1$ deficiency improves with age. This contrasts with children with most PIDs, whose clinical conditions typically deteriorate with time. Finally, Mendelian predisposition to a parasitic infection was recently documented in a patient with APOL1 deficiency who developed trypanosomiasis (45).

These investigations paved the way for the study of herpes simplex virus (HSV) encephalitis (HSE), the most common form of sporadic viral encephalitis in Western countries. Since the discovery of HSE in 1941, it has remained unclear why only a small fraction of otherwise healthy individuals exposed to HSV-1 develop HSE. Moreover, none of the known PIDs, including SCN, XLA, and SCID, increases the risk of HSE. Two genetic etiologies of HSE have recently been discovered: autosomal recessive unc 93 homolog B (UNC93B) deficiency (46) and autosomal dominant TLR3 deficiency (47). These disorders lead to impaired recognition of dsRNA intermediates of HSV-1 in the CNS, resulting in impaired type I and type III IFN production, which, in turn, results in enhanced viral replication and cell death. The virus does not spread to other organs because other cell types seem to be capable of controlling HSV-1 by TLR3-independent processes. Similarly, patients seem to use other means to control other viruses. The incomplete clinical penetrance probably reflects the influence of other factors, such as age at HSV-1 infection and modifier genes. In any event, it probably accounts for HSE being sporadic in most kindreds. The identification of a Mendelian basis for HSE provided the first demonstration that a sporadic, life-threatening infectious disease that had not been associated with any known inherited or acquired risk factor may result from a monogenic disorder, at least in some patients $(48,49)$. The field of Mendelian predisposition to infectious diseases thus covers an immense spectrum, ranging from profound cytopenia, in which patients are vulnerable to most (but not all) microbes, to HSE, in which leukocytes are apparently not involved in the immunodeficiency and otherwise healthy patients 
Table 1

Mendelian holes in immunity to infection

\begin{tabular}{|c|c|c|c|c|}
\hline Infectious agent & Clinical phenotype & Immunological phenotype & Gene & Refs. \\
\hline Neisseria & $\begin{array}{l}\text { Invasive disease } \\
\text { Invasive disease }\end{array}$ & $\begin{array}{c}\text { MAC deficiency } \\
\text { Properdin deficiency }\end{array}$ & $\begin{array}{c}\mathrm{C5}, \mathrm{C6}, \mathrm{C7}, \mathrm{C8A}, \mathrm{C} 8 \mathrm{~B}, \mathrm{C} 8 \mathrm{G}, \mathrm{C9} \\
\text { PFC }\end{array}$ & $\begin{array}{l}\text { Reviewed } \\
\text { in ref. } 16\end{array}$ \\
\hline Mycobacteria & $\begin{array}{c}\text { MSMD } \\
\text { Disseminated tuberculosis }\end{array}$ & IL-12/IL-23-IFN- $\gamma$ deficiency & $\begin{array}{c}\text { IFNGR1, IFNGR2, STAT1, } \\
\text { NEMO, IL12B, } \\
\text { IL12RB1 }\end{array}$ & $\begin{array}{l}\text { Reviewed } \\
\text { in ref. } 43\end{array}$ \\
\hline $\begin{array}{l}\text { Streptococcus } \\
\text { pneumoniae }\end{array}$ & Invasive disease & IRAK-4 and MyD88 deficiency & IRAK4, MYD88 & 37,38 \\
\hline EBV & X-linked lymphoproliferative disease & SAP and XIAP deficiency & $S A P, X I A P$ & 36,100 \\
\hline HPV & Epidermodysplasia verruciformis & EVER1/EVER2 deficiency & EVER1, EVER2 & 33 \\
\hline HSV-1 & Encephalitis & Impaired production of antiviral IFNs & UNC93B, TLR3 & 46,47 \\
\hline $\begin{array}{l}\text { Trypanosoma } \\
\text { evansi }\end{array}$ & Febrile episodes & No trypanolytic activity & APOL1 & 45 \\
\hline
\end{tabular}

C, complement component; MAC, membrane attack complex; NEMO, NF-kB essential modulator; PFC, properdin factor, complement.

are vulnerable to primary HSV-1 infection of the CNS. As in the case of individuals deficient for IL-12p 40 , IL-12R $\beta 1$, IRAK-4, or MyD88, the condition of children genetically susceptible to HSE improves with age. These children would typically not have been considered to have a PID in the early 1950s. However, the discovery of the Mendelian genetic basis of their conditions raises the possibility that more, if not most, severe infections in otherwise healthy children may result from PIDs. Candidate gene approaches, genome-wide linkage (GWL) studies, and even perhaps whole genome sequencing may soon reveal further Mendelian genetic etiologies for severe pediatric infectious diseases.

\section{Mendelian resistance}

Genetically determined resistance to an infectious agent is the obligate mirror of the genetically determined susceptibility phenotype. The term resistance typically applies in the setting of infections caused by virulent microbes. Virulent microbes are defined as those causing disease - often lethal disease - in most individuals of the population studied. Conversely, when most infected individuals do not develop disease, it is more common to speak of the susceptibility of the patients. To our knowledge, four Mendelian traits have been found to confer resistance to specific infections, as they result in a lack of the receptors used by the invading microbes (Table 2). Consequently, individuals carrying the common wild-type alleles are intrinsically susceptible to these particular pathogens, whereas individuals carrying the mutant alleles display almost complete and apparently specific protection against these pathogens. Protection against Plasmodium vivax, a pathogen that causes malaria, is conferred by a lack of Duffy blood group, chemokine receptor (DARC), a coreceptor for $P$. vivax, on erythrocytes (18). The resistance trait is recessive, and the single nucleotide mutation affects the GATA-1-binding site in the promoter of the DARC gene, selectively preventing gene transcription in erythroid cells (50). Recessive resistance to HIV-1 infection has been found to be conferred by mutations affecting the extracellular domain of another chemokine receptor, CCR5. CCR5 functions with CD4 as a coreceptor for HIV-1 on CD4 ${ }^{+} \mathrm{T}$ cells (51). Subjects homozygous for the most common deleterious CCR5 mutation, a 32-bp deletion $(\Delta 32)$, display strong protection against infection with CCR5-tropic HIV-1 (52-54). The eryth- rocyte $\mathrm{P}$ antigen is the cellular receptor for parvovirus $\mathrm{B} 19$. The small number of individuals with the p phenotype, whose erythrocytes do not have this receptor, are resistant to infection with this virus, which causes erythema infectiosum and severe forms of acute erythroblastopenic anemia in children with hemolytic anemia (55). Finally, resistance to noroviruses (also known as Norwalk-like viruses), a leading cause of gastroenteritis, was recently shown to be associated with mutations in the fucosyltransferase 2 (FUT2) gene (56), which encodes an $\alpha(1,2)$-fucosyltransferase that regulates the expression of $\mathrm{ABH}$ histo-blood group antigens on the surface of epithelial cells and in mucosal secretions (57). Several inactivating FUT2 mutations are responsible for the nonsecretor phenotype $\left(\mathrm{Se}^{-}\right)$characterized by a lack of expression of norovirus coreceptors, $\mathrm{ABH}$ antigens, on epithelial cells and complete resistance to symptomatic norovirus infection in experimental and natural conditions $(58,59)$. It is thus clear that infectious diseases caused by these four common pathogens result from autosomal dominant susceptibility. Mendelian predisposition to infectious diseases may therefore be common.

Given their obvious benefits, alleles conferring Mendelian resistance to virulent pathogens would be expected to be under strong positive selection pressure. This is probably the case for the DARC mutation, which is not found in Europe but has a frequency of up to $80 \%$ in African populations, in which P. vivax is endemic (18). Moreover, $P$. vivax was recently shown to be more virulent than previously thought $(60,61)$. However, this parasite was found to be transmitted in some populations with no DARC on their erythrocytes $(62,63)$. Therefore, DARC is probably not the only coreceptor for $P$. vivax, and its precise role in host resistance under natural conditions needs to be reevaluated (7). Similarly, variants of erythrocyte disease-causing genes conferring a major (but not Mendelian) resistance, as defined in the next section, against malaria caused by $P$. falciparum are much more common in countries where $P$. falciparum is endemic. The best example is provided by the worldwide spread of the deleterious $\beta$-hemoglobin $(H B B)$ allele known as hemoglobin S (HbS) (64). Homozygosity for this allele causes life-threatening sickle cell disease (drepanocytosis), but heterozygosity protects against severe malaria caused by $P$. falciparum (11). This results in heterosis, in which heterozygotes have a selective advantage over both types of homozygotes 
Table 2

Mendelian resistance to infection

$\begin{array}{ll}\text { Infectious agent } & \text { Clinical phenotype } \\ P . \text { vivax } & \text { Natural resistance } \\ \text { HIV-1 } & \text { Natural resistance } \\ \text { Norovirus } & \text { Natural resistance } \\ \text { Parvovirus } & \text { Natural resistance }\end{array}$

$\begin{array}{ccc}\text { Immunological phenotype } & \text { Gene } & \text { Refs. } \\ \text { Lack of coreceptor for pathogen } & \text { DARC } & 18 \\ \text { Lack of coreceptor for pathogen } & \text { CCR5 } & 52-54 \\ \text { Lack of coreceptor for pathogen } & \text { FUT2 } & 56,58 \\ \text { Lack of coreceptor for pathogen } & ? & 55\end{array}$

the designation as major genes of loci identified by means of linkage studies in the context of genome-wide screening. Indeed, the loci detected in GWL scans, particularly in those studies based on the commonly used "affected sib-pairs" design, should have a substantial influence on the phenotype under study that may be qualified as

(65). By contrast, the $\mathrm{HbC}$ allele confers recessive, but not dominant, resistance against malaria caused by P. falciparum, possibly accounting for the limited spread of this allele in one geographical region (West Africa) (66). The first population genetics studies of CCR5 found that the main resistance allele, CCR $5^{\Delta 32}$, originated from a single ancestor of European origin (67). The relatively recent estimated date of the mutation event (approximately 2,000-3,000 years ago), the frequency of the allele in the European population (10\% in Western and Central Europe), and the long-range linkage disequilibrium pattern at the CCR5 locus are highly suggestive of positive selection (68). However, the intensity (69) and nature (51) of the selective pressure remain to be determined. The situation may be even more complicated, as homozygosity for the CCR $5^{\Delta 32}$ allele was recently reported to be associated with symptomatic West Nile virus infection (70), indicating that selective pressure may also be negative, depending on the microbial environment. Finally, a single nonsense FUT2 mutation, G428A, is the most common mutation responsible for the $\mathrm{Se}^{-}$phenotype in populations of European and African descent, representing more than $95 \%$ of the European null FUT2 alleles $(59,71)$. It remains unclear whether the recurrence of this mutation is due to a hot spot and/or a founder effect under positive selective pressure. Mendelian resistance genes have thus provided the best illustrations of natural selection on the human genome. Many other human mutations probably have been or are being selected because they confer Mendelian resistance to virulent pathogens. In any event, these four disorders have revolutionized our perception of common infections, as they provide proof of principle that common infectious illnesses may also be truly Mendelian traits.

\section{Major genes}

The "major gene/locus" concept was developed in the 1960s, decades after the introduction of the polygenic model of inheritance in 1918 by Ronald Fisher (72). This concept was developed at a time when clinical geneticists needed a framework that could explicitly specify the effect of a single gene on the expression of a disease $(73,74)$. A major gene differs from a Mendelian effect in displaying lower penetrance, due to a greater influence of both other genes and environmental factors in the individual. This concept was first formalized and developed in the context of complex segregation analysis, which is a statistical method based on a model of inheritance in which a given phenotype may result from the joint effects of a major locus, a polygenic component, and environmental factors $(74,75)$. Since the 1970 s, several major genes identified by segregation analyses have been reported in a number of complex traits, including infectious disease-related phenotypes in leprosy, malaria, schistosomiasis, and some viral infections (4). The development of highly polymorphic genetic markers has recently led to a major effect $(76,77)$. Several such major loci have been mapped in infectious diseases (4). The first was mapped to chromosome $5 q 31-q 33$ and controls levels of infection with the parasite Schistosoma mansoni, the agent of schistosomiasis (78). The most recent was mapped to chromosome $8 \mathrm{q} 12$ and confers predisposition to pulmonary tuberculosis (79). These two chromosomal regions are large, and the actual major susceptibility loci await precise identification. The major gene concept may evolve further, particularly in the context of technological advances in genomics, such as the development of genome-wide association (GWA) studies. Furthermore, major genes may be specific to a given population (e.g., those defined by ethnic origin, history, or age at onset of disease) and, for an infectious disease (e.g., leprosy), may be specific for a given immunological or clinical phenotype (e.g., paucibacillary leprosy).

The HbS trait may be considered the first major gene identified in a common infectious disease (11), based on both its frequency in some African populations and its effect on severe malaria (64). No GWL screen has been conducted for severe malaria, probably because of the rarity of this clinical phenotype and the even greater rarity of families with multiple cases. However, two recent GWA studies suggest that $H B B$ is the strongest genetic determinant of resistance to severe malaria (R. Horstman and D. Kwiatkowski, personal communications). As results for malaria require confirmation, leprosy is currently the only infectious disease for which a complete successful positional cloning approach -including GWL screening, followed by refined linkage disequilibrium mapping - has led to the identification of major susceptibility variants. Leprosy is a chronic infectious disease caused by Mycobacterium leprae; it continues to affect more than 300,000 new individuals per year (80). Both the development of leprosy per se upon exposure to $M$. leprae and its spectrum of clinical features depend on human genes $(42,81)$. The first evidence for this was provided by twin studies carried out in the 1960s, followed by several segregation studies, most detecting the presence of a major gene (82, 83). Three major genes were recently mapped by GWL studies. The first of these studies focused on paucibacillary leprosy in India and detected a major locus on chromosome 10p13 (84) that has yet to be precisely identified. The second was carried out in Vietnam and mapped a major gene for susceptibility to leprosy per se to chromosome 6q25 (85). Further linkage disequilibrium studies identified variants of the regulatory region shared by PARK2, a gene encoding the E3-ubiquitin ligase Parkin, and PACRG (Parkin coregulated gene) as genetic risk factors for leprosy (86). In addition to identifying a novel pathway of immunity to M. leprae (87), not predicted by any previous experimental investigation in animal models, this study was the first to report successful positional cloning of a major locus in a common infectious disease. Finally, a major locus associated with predisposition to early-onset leprosy was recently identified in the same Vietnamese sample. A single 
functional polymorphism in the lymphotoxin- $\alpha$ (LTA) gene was shown to have a strong impact on the risk of developing leprosy before the age of 25 years (88). Further GWL and perhaps GWA studies are expected to reveal additional major genes predisposing to common infectious diseases.

\section{Multigenic predisposition}

It is common to distinguish two patterns of multigenic inheritance - oligogenic and polygenic - according to the number and relative impact of the genes influencing disease. Oligogenicity implies that the phenotype is dependent on two or a few major genes, with other genetic factors making a relatively lower contribution. Polygenicity implies that the phenotype results from the effect of a large number of genetic loci, each having a small effect. As discussed above, there is no unambiguous definition of major gene, and it is even possible to use a mixture of definitions. Indeed, it would be legitimate to consider tuberculosis to be an example of an oligogenic disease (one major gene on $8 q 12$ identified by GWL analysis [ref. 79] and another gene, natural resistance-associated macrophage protein 1 [NRAMP1], identified from candidate gene association and linkage analysis [refs. 89-91]) if both genes were found to affect the same clinical phenotype in the same population sample, but this was not actually the case. We pointed out above that three major genes conferring a predisposition to leprosy have been identified, on chromosomes $10 \mathrm{p} 13,6 \mathrm{q} 25$, and $6 \mathrm{p} 21$. Two of these genes (PARK2 and LTA) were shown to affect the same phenotype, leprosy per se, in the same Vietnamese sample, with a stronger effect in early-onset cases (ref. 88 and our unpublished observations). The study of genetic predisposition to leprosy has therefore led not only to the first successful positional cloning of a major locus in a common infectious disease, but also to the first demonstration of an oligogenic predisposition to a common infectious disease, at least at the population level. An implicit but fundamental idea underlying multigenic inheritance is that, mechanistically, oligogenic and polygenic modes of inheritance apply to individuals. As this idea of a cumulative effect of the genes at the individual level is implicit, this point is often overlooked, and it is common practice to describe multigenic predisposition at the population level. However, multigenic predisposition at the population level does not necessarily reflect multigenic inheritance at the individual level, as genetic or even phenotypic heterogeneity may be involved, with certain genes acting as major genes only in certain individuals or groups of individuals in the population considered.

The identification of a truly polygenic predisposition requires a large number of individuals, both because of the small expected effect attributable to each gene and because of the additive nature of these genetic effects. It is therefore not surprising that the proof of principle of such genetic mechanisms at both the population and individual levels has not yet been provided by human studies, but only by studies of susceptibility to infectious diseases in animal models of experimental infections. As a textbook example, it has been shown in a murine model of malaria caused by infection with Plasmodium chabaudi AS that at least five loci control parasitemia and nine control survival (14). Several genes have often been reported to have a potential influence on the onset of a given infectious disease in humans, but there is currently no proof of polygenic predisposition per se in specific populations and individual humans. For example, a number of genes (e.g., HLA-DR loci, NRAMP1, and IL12RB1) have been reported to play a role in tuberculosis, but it has never been determined whether these genes act independently and additively on the same phenotype in the same sample. Despite these limitations, references to the polygenic nature of predisposition to a human infectious disease have been made at the population level. We are even more ignorant of the genetic determinism at individual level. However, with the advent of GWA studies, by capturing a large proportion of the genetic variation through the genotyping of millions of SNPs, in thousands of cases and controls, we may be able to detect polygenic predisposition in individuals and populations. The first GWA study on infectious disease has recently been published (92) and seems to indicate that several polymorphisms (e.g., HLA alleles) are involved in the genetic control of HIV-1 viral load, accounting for almost $15 \%$ of the phenotypic variation observed in asymptomatic individuals. More recent GWA studies on severe malaria identified $H B B$ as the major signal of association (R. Horstman and D. Kwiatkowski, personal communications), although previous epidemiological studies of African children implied that $H B B$ makes only a minor contribution to the total impact of host genetics in hospitalized patients with malaria (93). Discovering these other genetic factors by GWA requires larger sample sizes and higher SNP density due to the low linkage disequilibrium in African populations. Future GWA studies on other infectious diseases, such as tuberculosis, should help us address the question of the multigenic architecture of complex infectious diseases.

\section{Concluding remarks}

Genetic investigations began in the early 1950s, but proof of principle for various complementary types of genetic predisposition was obtained only in the last decade, unifying hitherto separate lines of investigation (94). Conventional PIDs have been studied in most detail over the last 50 years. Association studies have so far identified only a handful of convincing infection susceptibility/resistance alleles (e.g., HbS). Linkage studies have recently identified a set of major genes (in the case of leprosy), and novel PIDs have provided a Mendelian basis for predisposition to certain infectious diseases (e.g., HSE), bridging the gap between the two fields dealing with genetic predisposition to infection. Much progress is expected in these two fields in the near future. We believe there are also more Mendelian resistance genes to be discovered. There is therefore probably considerable human genetic determinism in the pathogenesis of infectious diseases. Indeed, we believe that infectious diseases are largely genetically determined, probably more so than most other human diseases. The increase in life expectancy observed in the 20th century occurred despite the retention in the genomes of most individuals of genes conferring poor immunity to particular infectious agents (94). There has been no sudden natural selection of high-quality immune system genes worldwide. This persistent immunodeficiency has simply been masked by medical progress, in the form of improved hygiene, vaccines, and antibiotics. The genetic theory of infectious diseases does not conflict with the microbial theory of these diseases, and Louis Pasteur himself stated in his seminal survey of diseases affecting silk worms, that in the course of "flacherie," which he designated as "hereditary," "it is not the microbe that is transmitted from the parents to the offspring, but the predisposition to disease" (95). This theory accounts for inter-individual variability in the course of infection, by providing an explanation of the pathogenesis of infection. However, only a very small fraction of human infectious diseases are understood at the genetic 
level, and in only a few patients. The field of the human genetics of infectious diseases now aims to build on this fragile proof of principle to establish a solid paradigm shift. It is necessary to demonstrate that the findings of previous studies constitute the rule rather than an exception.

The thorough dissection of increasing numbers of infectious diseases will confront investigators with the intriguing question of the genetic architecture of infectious diseases. In addition to the identification of new genes, one key question concerns the definition of the proportion of Mendelian (typically, but not necessarily, rare alleles) and more complex (typically, but not necessarily, common alleles) predisposition and resistance in individuals and populations $(96,97)$. Needless to say, there is clearly no such thing as a strict Mendelian segregation of phenotypes, because no singlegene organism exists. All phenotypes are therefore multigenic in essence. It is, however, important to define the hierarchy of the genes involved in predisposition to infection in both individuals and populations. There may be a general pattern of genetic predisposition and resistance, a predictable genetic architecture accounting for most, if not all, clinical situations. Traditionally, the two parameters that have been taken into account when reflecting on the genetic basis of infectious diseases in general terms were the frequency of the disease (rare versus common infections) and the general resistance of the infected individual (susceptible to multiple infections versus normally resistant to other infections). The dichotomy between PIDs (rare conditions, with one gene affected, conferring predisposition to multiple infections) and polygenic inheritance (common predisposition, with multiple genes affected, conferring predisposition to a single infection in otherwise healthy children) collapsed with the identification of novel PIDs and major genes in individuals and populations, respectively. We suggest that age at infection (before or after puberty) and the potential severity of infection (deaths rare or common in the absence of therapeutic intervention) may be more useful parameters for definition of the genetic predisposition to an infectious disease. More specifically, our working hypothesis, which is consistent with the available data, is that primary infections have a strong Mendelian component (typically, but not exclusively in children, and typically, but not necessarily, due to rare alleles), conferring Mendelian susceptibility and resistance to rare and common severe diseases. By contrast, the corresponding infectious diseases occurring in the course of secondary infection or reactivation from latency probably result from a more complex predisposition (typically, but not necessarily, due to common alleles).

The immunological implications of the human genetics of infectious diseases are considerable, particularly for Mendelian studies, which provide an ideal way of defining the function of immune system genes in natura, i.e., in the setting of a natural ecosystem (5,
98, 99). Clinical studies are often considered to benefit from basic scientific studies, and they certainly do. Clinical investigations, in the field of human genetics in particular, provide us with unique opportunities to explore "experiments of nature" (99), thereby defining the physiological and pathological roles of cells and molecules in natural conditions. This field also has major clinical implications, with the discovery of Mendelian traits most likely to be of benefit to patients. Studies of the human genetics of infectious diseases promise to provide patients with a genetic diagnosis, which may follow the microbiological diagnosis, paving the way for the genetic counseling of affected families. This research has other clinical implications, in terms of the development of new therapeutic interventions, such as the life-saving effects of G-CSF in patients with SCN; of IgG in patients with XLA; of HSCT and gene therapy in patients with SCID; and also of exogenous IFN- $\gamma$ and IFN- $\alpha$ treatment in patients with mycobacterial disease and HSE, respectively. This field is also likely to revolutionize the treatment of infectious diseases, with efforts made to restore or to enhance immune system capacity, in line with the seminal breakthroughs of 19th century immunology - vaccination and serotherapy - rather than to kill infectious agents directly. The discovery of sulfamides and antibiotics in the 1930s, and their widespread use from the 1940s onward, have not ended the search for immunostimulatory drugs to combat infectious agents. The use of G-CSF, IgG, and HSCT followed the description of immunological lesions underlying severe infections. Free of any immunological bias, GWL and GWA studies, and perhaps soon whole genome sequencing, may reveal completely novel therapeutic avenues. Human genetics will increasingly provide a strong rationale for the development of new treatments, infection by infection and patient by patient, by identifying the missing component in a given individual patient's immunity to infection.

\section{Acknowledgments}

We thank the members of the Necker and Rockefeller branches of the Laboratory of Human Genetics of Infectious Diseases. We also thank Anthony Allison, Jonathan Howard, Rolf Horstman, and Dominic Kwiatkowski. Our research is funded by the Rockefeller University, Paris Descartes University, INSERM, Agence Nationale pour la Recherche, Institut Pasteur, the European Union, the Ordre de Malte, the Howard Hughes Medical Institute, the March of Dimes, the Dana Foundation, and the Gates Foundation.

Address correspondence to: Jean-Laurent Casanova, Laboratory of Human Genetics of Infectious Diseases, The Rockefeller University, 1230 York Avenue, Box 163, New York, New York 10065, USA. Phone: (212) 327-7331; Fax: (212) 327-7330; E-mail: jean-laurent. casanova@rockefeller.edu.
1. Pasteur, L. 1939. Oewvres de Pasteur, réunies et annotées par Louis Pasteur Vallery-Radot. Masson et Cie. Paris, France.

2. Nicolle, C. 1937. Destin des maladies infectieuses. Alcan. Paris, France. 301 pp.

3. Dubos, R.J. 1950. Lonis Pasteur, free lance of science. Little, Brown. Boston, Massachusetts, USA. 418 pp.

4. Casanova, J.L., and Abel, L. 2007. Human genetics of infectious diseases: a unified theory. $E M B O J$. 26:915-922.

5. Casanova, J.L., and Abel, L. 2004. The human model: a genetic dissection of immunity to infection in natural conditions. Nat. Rev. Immunol. 4:55-66.

6. Kwiatkowski, D. 2000. Science, medicine, and the future: susceptibility to infection. $B M J$. 321:1061-1065.

7. Allison, A. 2009. Observational, hypothesis-driven and genomics research strategies for analyzing inherited differences in responses to infectious diseases. Public Health Genomics. 12:41-52.

8. Alcais, A., and Abel, L. 2004. Application of genetic epidemiology to dissecting host susceptibility/ resistance to infection illustrated with the study of common mycobacterial infections. In Susceptibility to infectious diseases: the importance of host genetics. $\mathrm{R}$. Bellamy, editor. Cambridge University Press. Cambridge, United Kingdom/New York, New York, USA. 7-44.
9. Sorensen, T.I., Nielsen, G.G., Andersen, P.K., and Teasdale, T.W. 1988. Genetic and environmental influences on premature death in adult adoptees. N. Engl. J. Med. 318:727-732.

10. Bruton, O.C. 1952. Agammaglobulinemia. Pediatrics. 9:722-728.

11. Allison, A.C. 1954. Protection afforded by sickle cell trait against subtertian malarian infection. Br. Med. J. 1:290-294.

12. Notarangelo, L., et al. 2006. Primary immunodeficiency diseases: an update from the International Union of Immunological Societies Primary Immunodeficiency Diseases Classification Committee Meeting in Budapest, 2005. J. Allergy Clin. Immunol. 
117:883-896.

13. Ochs, H., Smith, C.I.E., and Puck, J. 2006. Primary immunodeficiencies: a molecular and genetic approach. Oxford University Press. New York, New York, USA. 776 pp.

14. Fortin, A., Stevenson, M.M., and Gros, P. 2002. Complex genetic control of susceptibility to malaria in mice. Genes Immun. 3:177-186.

15. Casanova, J.L., et al. 2005. From idiopathic infectious diseases to novel primary immunodeficiencies. J. Allergy Clin. Immunol. 116:426-430.

16. Picard, C., Casanova, J.L., and Abel, L. 2006. Mendelian traits that confer predisposition or resistance to specific infections in humans. Curr. Opin. Immunol. 18:383-390.

17. Barnwell, J.W., Nichols, M.E., and Rubinstein, P. 1989. In vitro evaluation of the role of the Duffy blood group in erythrocyte invasion by Plasmodium vivax. J. Exp. Med. 169:1795-1802.

18. Miller, L.H., Mason, S.J., Clyde, D.F., and McGinniss, M.H. 1976. The resistance factor to Plasmodium vivax in blacks. The Duffy-blood-group genotype, FyFy. N. Engl. J. Med. 295:302-304.

19. Abel, L., and Casanova, J.L. 2000. Genetic predisposition to clinical tuberculosis: bridging the gap between simple and complex inheritance. Am.J. Hum. Genet. 67:274-277.

20. Bruton, O.C. 1962. A decade with agammaglobulinemia. J. Pediatr. 60:672-676.

21. Boztug, K., Welte, K., Zeidler, C., and Klein, C. 2008. Congenital neutropenia syndromes. Immunol. Allergy Clin. North Am. 28:259-275, vii-viii.

22. Conley, M.E., et al. 2005. Genetic analysis of patients with defects in early B-cell development. Immunol. Rev. 203:216-234.

23. Fischer, A., et al. 2005. Severe combined immunodeficiency. A model disease for molecular immunology and therapy. Immunol. Rev. 203:98-109.

24. Bonilla, M.A., et al. 1989. Effects of recombinant human granulocyte colony-stimulating factor on neutropenia in patients with congenital agranulocytosis. N. Engl. J. Med. 320:1574-1580.

25. Gatti, R.A., Meuwissen, H.J., Allen, H.D., Hong, R., and Good, R.A. 1968. Immunological reconstitution of sex-linked lymphopenic immunological deficiency. Lancet. 2:1366-1369.

26. Good, R.A. 1987. Bone marrow transplantation for immunodeficiency diseases. Am. J. Med. Sci. 294:68-74.

27. Cavazzana-Calvo, M., et al. 2000. Gene therapy of human severe combined immunodeficiency (SCID)-X1 disease. Science. 288:669-672.

28. Hacein-Bey-Abina, S., et al. 2003. LMO2-associated clonal $\mathrm{T}$ cell proliferation in two patients after gene therapy for SCID-X1. Science. 302:415-419.

29. Hacein-Bey-Abina, S., et al. 2008. Insertional oncogenesis in 4 patients after retrovirus-mediated gene therapy of SCID-X1. J. Clin. Invest. 118:3132-3142.

30. Gaspar, H.B., et al. 2004. Gene therapy of X-linked severe combined immunodeficiency by use of a pseudotyped gammaretroviral vector. Lancet. 364:2181-2187.

31. Howe, S.J., et al. 2008. Insertional mutagenesis combined with acquired somatic mutations causes leukemogenesis following gene therapy of SCID$\mathrm{X} 1$ patients. J. Clin. Invest. 118:3143-3150.

32. Orth, G. 2008. Host defenses against human papillomaviruses: lessons from epidermodysplasia verruciformis. Curr. Top. Microbiol. Immunol. 321:59-83.

33. Ramoz, N., et al. 2002. Mutations in two adjacent novel genes are associated with epidermodysplasia verruciformis. Nat. Genet. 32:579-581.

34. Lazarczyk, M., et al. 2008. Regulation of cellular zinc balance as a potential mechanism of EVERmediated protection against pathogenesis by cutaneous oncogenic human papillomaviruses. J. Exp. Med. 205:35-42.

35. Purtilo, D.T., Cassel, C.K., Yang, J.P., and Harper, R.
1975. X-linked recessive progressive combined variable immunodeficiency (Duncan's disease). Lancet. 1:935-940

36. Rigaud, S., et al. 2006. XIAP deficiency in humans causes an X-linked lymphoproliferative syndrome. Nature. 444:110-114.

37. Picard, C., et al. 2003. Pyogenic bacterial infections in humans with IRAK-4 deficiency. Science. 299:2076-2079.

38. von Bernuth, H., et al. 2008. Pyogenic bacterial infections in humans with MyD88 deficiency. Science. 321:691-696.

39. Ku, C.L., et al. 2007. Selective predisposition to bacterial infections in IRAK-4-deficient children: IRAK4-dependent TLRs are otherwise redundant in protective immunity. J. Exp. Med. 204:2407-2422.

40. Jouanguy, E., et al. 1996. Interferon-gammareceptor deficiency in an infant with fatal bacille Calmette-Guerin infection. N. Engl. J. Med. 335:1956-1961.

41. Newport, M.J., et al. 1996. A mutation in the interferon-gamma-receptor gene and susceptibility to mycobacterial infection. N. Engl. J. Med. 335:1941-1949.

42. Casanova, J.L., and Abel, L. 2002. Genetic dissection of immunity to mycobacteria: the human model. Annu. Rev. Immunol. 20:581-620.

43. Filipe-Santos, O., et al. 2006. Inborn errors of IL-12/23- and IFN-gamma-mediated immunity: molecular, cellular, and clinical features. Semin. Immunol. 18:347-361.

44. Fieschi, C., et al. 2003. Low penetrance, broad resistance, and favorable outcome of interleukin 12 receptor beta 1 deficiency: medical and immunological implications. J. Exp. Med. 197:527-535.

45. Vanhollebeke, B., et al. 2006. Human Trypanosoma evansi infection linked to a lack of apolipoprotein L-I. N. Engl. J. Med. 355:2752-2756

46. Casrouge, A., et al. 2006. Herpes simplex virus encephalitis in human UNC-93B deficiency. Science. 314:308-312.

47. Zhang, S.-Y., et al. 2007. TLR3 deficiency in otherwise healthy patients with herpes simplex encephalitis. Science. 317:1522-1527.

48. Sancho-Shimizu, V., et al. 2007. Genetic susceptibility to herpes simplex virus 1 encephalitis in mice and humans. Curr. Opin. Allergy Clin. Immunol. 7:495-505.

49. Zhang, S.Y., et al. 2007. Human Toll-like receptordependent induction of interferons in protective immunity to viruses. Immunol. Rev. 220:225-236.

50. Tournamille, C., Colin, Y., Cartron, J.P., and Le Van Kim, C. 1995. Disruption of a GATA motif in the Duffy gene promoter abolishes erythroid gene expression in Duffy-negative individuals. Nat. Genet. 10:224-228.

51. Arenzana-Seisdedos, F., and Parmentier, M. 2006. Genetics of resistance to HIV infection: role of coreceptors and co-receptor ligands. Semin. Immunol. 18:387-403.

52. Dean, M., et al. 1996. Genetic restriction of HIV1 infection and progression to AIDS by a deletion allele of the CKR5 structural gene. Science. 273:1856-1862.

53. Liu, R., et al. 1996. Homozygous defects in HIV-1 coreceptor accounts for resistance of some multiply-exposed individuals to HIV-1 infection. Cell. 86:367-377.

54. Samson, M., et al. 1996. Resistance to HIV-1 infection in Caucasian individuals bearing mutant alleles of teh CCR5 chemokine receptor gene. Nature. 382:722-725.

55. Brown, K.E., et al. 1994. Resistance to parvovirus B19 infection due to lack of virus receptor (erythrocyte P antigen). N. Engl. J. Med. 330:1192-1196.

56. Le Pendu, J., Ruvoen-Clouet, N., Kindberg, E., and Svensson, L. 2006. Mendelian resistance to human norovirus infections. Semin. Immunol. 18:375-386.
57. Marionneau, S., et al. 2002. Norwalk virus binds to histo-blood group antigens present on gastroduodenal epithelial cells of secretor individuals. Gastroenterology. 122:1967-1977.

58. Lindesmith, L., et al. 2003. Human susceptibility and resistance to Norwalk virus infection. Nat. Med. 9:548-553.

59. Thorven, M., et al. 2005. A homozygous nonsense mutation (428G-->A) in the human secretor (FUT2) gene provides resistance to symptomatic norovirus (GGII) infections. J. Virol. 79:15351-15355.

60. Genton, B., et al. 2008. Plasmodium vivax and mixed infections are associated with severe malaria in children: a prospective cohort study from Papua New Guinea. PLoS Med. 5:e127.

61. Tjitra, E., et al. 2008. Multidrug-resistant Plasmodium vivax associated with severe and fatal malaria: a prospective study in Papua, Indonesia. PLoS Med. 5:e128.

62. Cavasini, C.E., et al. 2007. Duffy blood group gene polymorphisms among malaria vivax patients in four areas of the Brazilian Amazon region. Malar. J. 6:167.

63. Ryan, J.R., et al. 2006. Evidence for transmission of Plasmodium vivax among a duffy antigen negative population in Western Kenya. Am. J. Trop. Med. Hyg. 75:575-581.

64. Kwiatkowski, D.P. 2005. How malaria has affected the human genome and what human genetics can teach us about malaria. Am. J. Hum. Genet. 77:171-192.

65. Vogel, F., and Motulsky, A.G. 1997. Human genetics: problems and approaches. Springer. Berlin, Germany/ New York, New York, USA. 851 pp.

66. Modiano, D., et al. 2001. Haemoglobin C protects against clinical Plasmodium falciparum malaria. Nature. 414:305-308.

67. Stephens, J.C., et al. 1998. Dating the origin of the CCR5-Delta32 AIDS-resistance allele by the coalescence of haplotypes. Am. J. Hum. Genet. 62:1507-1515.

68. Novembre, J., Galvani, A.P., and Slatkin, M. 2005. The geographic spread of the CCR5 Delta32 HIVresistance allele. PLoS Biol. 3:e339.

69. Sabeti, P.C., et al. 2005. The case for selection at CCR5-Delta32. PLoS Biol. 3:e378

70. Glass, W.G., et al. 2006. CCR5 deficiency increases risk of symptomatic West Nile virus infection. J. Exp. Med. 203:35-40.

71. Liu, Y., et al. 1998. Extensive polymorphism of the FUT2 gene in an African (Xhosa) population of South Africa. Hum. Genet. 103:204-210.

72. Fisher, R.A. 1918. The correlation between relatives on the supposition of Mendelian inheritance. Trans. R. Soc. Edinb. 52:399-433.

73. Edwards, J.H. 1969. Familial predisposition in man. Br. Med. Bull. 25:58-64.

74. Lalouel, J.M., Rao, D.C., Morton, N.E., and Elston, R.C. 1983. A unified model for complex segregation analysis. Am. J. Hum. Genet. 35:816-826.

75. Khoury, M.J., Beaty, T.H., and Cohen, B.H. 1993. Fundamentals of genetic epidemiology. Oxford University Press. New York, New York, USA. 383 pp.

76. Risch, N. 1990. Linkage strategies for genetically complex traits. III. The effect of marker polymorphism on analysis of affected relative pairs. Am.J. Hum. Genet. 46:242-253.

77. Risch, N., and Merikangas, K. 1996. The future of genetic studies of complex human diseases. Science. 273:1516-1517

78. Marquet, S., et al. 1996. Genetic localization of a locus controlling the intensity of infection by Schistosoma mansoni on chromosome 5q31-q33. Nat. Genet. 14:181-184.

79. Baghdadi, J.E., et al. 2006. An autosomal dominant major gene confers predisposition to pulmonary tuberculosis in adults. J. Exp. Med. 203:1679-1684. 80. WHO. 2006. Global leprosy situation, beginning of 


\section{review series}

2008. Wkly. Epidemiol. Rec. 83:293-300

81. Alcais, A., Mira, M., Casanova, J.L., Schurr, E., and Abel, L. 2005. Genetic dissection of immunity in leprosy. Curr. Opin. Immunol. 17:44-48.

82. Abel, L., and Demenais, F. 1988. Detection of major genes for susceptibility to leprosy and its subtypes. Am. J. Hum. Genet. 42:256-266.

83. Abel, L., et al. 1995. Complex segregation analysis of leprosy in southern Vietnam. Genet. Epidemiol. 12:63-82.

84. Siddiqui, M.R., et al. 2001. A major susceptibility locus for leprosy in India maps to chromosome 10p13. Nat. Genet. 27:439-441.

85. Mira, M.T., et al. 2003. Chromosome 6q25 is linked to susceptibility to leprosy in a Vietnamese population. Nat. Genet. 33:412-415.

86. Mira, M.T., et al. 2004. Susceptibility to leprosy is associated with PARK2 and PACRG. Nature. 427:636-640.

87. Schurr, E., Alcais, A., de Leseleuc, L., and Abel, L. 2006. Genetic predisposition to leprosy: a major gene reveals novel pathways of immunity to Myco- bacterium leprae. Semin. Immunol. 18:404-410.

88. Alcais, A., et al. 2007. Stepwise replication identifies a low-producing lymphotoxin-alpha allele as a major risk factor for early-onset leprosy. Nat. Genet. 39:517-522

89. Bellamy, R., et al. 1998. Variations in the NRAMP1 gene and susceptibility to tuberculosis in West Africans. N. Engl. J. Med. 338:640-644.

90. Greenwood, C.M., et al. 2000. Linkage of tuberculosis to chromosome 2q35 loci, including NRAMP1, in a large aboriginal Canadian family. Am. J. Hum. Genet. 67:405-416.

91. Malik, S., et al. 2005. Alleles of the NRAMP1 gene are risk factors for pediatric tuberculosis disease. Proc. Natl. Acad. Sci. U. S. A. 102:12183-12188.

92. Fellay, J., et al. 2007. A whole-genome association study of major determinants for host control of HIV-1. Science. 317:944-947.

93. Mackinnon, M.J., Mwangi, T.W., Snow, R.W., Marsh, K., and Williams, T.N. 2005. Heritability of malaria in Africa. PLoS Med. 2:e340.

94. Casanova, J.L., and Abel, L. 2005. Inborn errors of immunity to infection: the rule rather than the exception. J. Exp. Med. 202:197-201.

95. Pasteur, L. 1870. Etudes sur la maladie des vers a soie. La pébrine et la flacherie (tome I). Gauthier-Villars. Paris, France. 282 pp.

96. Antonarakis, S.E., and Beckmann, J.S. 2006. Mendelian disorders deserve more attention. Nat. Rev. Genet. 7:277-282.

97. Pritchard, J.K. 2001. Are rare variants responsible for susceptibility to complex diseases? Am. J. Hum. Genet. 69:124-137.

98. Casanova, J.L., and Abel, L. 2007. Primary immunodeficiencies: a field in its infancy. Science. 317:617-619.

99. Quintana-Murci, L., Alcais, A., Abel, L., and Casanova, J.L. 2007. Immunology in natura: clinical, epidemiological and evolutionary genetics of infectious diseases. Nat. Immunol. 8:1165-1171.

100.Coffey, A.J., et al. 1998. Host response to EBV infection in X-linked lymphoproliferative disease results from mutations in an SH2-domain encoding gene. Nat. Genet. 20:129-135. 